Charlemagne's challenge: the periodic latency problem

Sofie Coene, Frits C.R. Spieksma and Gerhard J. Woeginger

DEPARTMENT OF DECISION SCIENCES AND INFORMATION MANAGEMENT (KBI) 


\title{
Charlemagne's challenge: the Periodic Latency Problem
}

\author{
Sofie Coene* Frits C.R. Spieksma* Gerhard J. Woeginger ${ }^{\dagger}$
}

\begin{abstract}
Latency problems are characterized by their focus on minimizing the waiting time for all clients. We study periodic latency problems, a nontrivial extension of standard latency problems. In a periodic latency problem each client has to be visited regularly: there is a server traveling at unit speed, and there is a set of $n$ clients with given positions. The server must visit the clients over and over again, subject to the constraint that successive visits to client $i$ are at most $q_{i}$ time units away from each other.

We investigate two main problems. In problem PLPP the goal is to find a repeatable route for the server visiting as many clients as possible, without violating their $q_{i}$ 's. In problem PLP the goal is to minimize the number of servers needed to serve all clients. In dependence on the topology of the underlying network, we derive polynomial-time algorithms or hardness results for these two problems. Our results draw sharp separation lines between easy and hard cases.
\end{abstract}

Key words: latency problem, periodicity, complexity

\section{Prologue}

During his reign in the years 768-814, Charlemagne traveled constantly through his empire in Western Europe. Counts had been appointed to govern different pieces of Charlemagne's empire (called counties). On his travels, Charlemagne visited his counts regularly. One reason for these visits was to ensure loyalty of his counts. Indeed, when a count was not visited for a certain period, the count would no longer obey Charlemagne, and declare independence, thereby rising against the emperor. Clearly, this would force Charlemagne to act, and start an expensive war against the rebeling count. Charlemagne's challenge was to find a visiting sequence of his counts so that the time elapsed between two consecutive visits to a count would not exceed the "loyalty period" of that count.

\section{Introduction}

Consider the following problem. We are given a set of customers $N=\{1,2, \ldots, n\}$ with their positions $x_{1}, x_{2}, \ldots, x_{n}$ in some metric space; for each pair of cus-

\footnotetext{
* Katholieke Universiteit Leuven, Operations Research Group, Naamsestraat 69, B-3000 Leuven, Belgium.

${ }^{\dagger}$ Department of Mathematics, TU Eindhoven, P.O. Box 513, 5600 MB Eindhoven, The Netherlands
} 
tomers $i, j \in N$, there is a distance $d_{i j}$. We are also given a server that travels at unit speed (and always at full speed). There is a number $q_{i}$ associated with every customer $i$ which indicates the periodicity of customer $i, i \in N$. More precisely, $q_{i}$ is the maximal amount of time that is allowed to pass between two consecutive visits to customer $i, i \in N$. Each customer $i \in N$ also has an associated profit $p_{i}$. A customer $i$ is called served when the time elapsed between each two consecutive visits does not exceed $q_{i}, i \in N$. The goal is to find a travel-plan for the server which maximizes the total profit of the customers served. This travel plan can be represented by a list of customers (of infinite length) that prescribes the sequence in which the served customers are visited. Thus, in a feasible solution, (i) each served customer is visited an infinite number of times, and (ii) the time elapsed between two consecutive visits to customer $i$ does not exceed $q_{i}, i \in N$. We assume that all data are integral. We call this problem the Periodic Latency Problem with Profits (PLPP).

Clearly, referring back to Charlemagne's challenge, a count is a customer, Charlemagne is the server, and the loyalty periods are represented by the $q_{i}$ 's. If the profit of a customer represents the area of the county, Charlemagne's challenge is to maximize the size of his empire without having to fight internal wars.

In this paper, we also consider the problem where multiple servers are available and all customers need to be served. We assume here that a customer must be served by a single server; see Section 5 for the relevance of this assumption. The goal is then to minimize the number of servers required to serve all customers periodically. We call this problem the Periodic Latency Problem (PLP). Further, we dedicate a short section to the periodic latency problem with profits and multiple servers (MPLPP). Here again the goal is to find routes for the servers such that total collected profit is maximized.

\section{Why 'latency'?}

Latency problems are characterized by their focus on total waiting time as an objective function, see e.g. de Paepe et al. [2004] and the references contained therein. Latency problems differ from problems where travel time of the server is the objective. Notice that the problems that we study here, share the same fundamental property with latency problems: any period in time matters to all customers. That is why we refer to the problems described here as periodic latency problems; indeed, time matters for the customers, whereas the distance traveled by the server is of no interest.

\section{Motivation}

We see the PLPP and the PLP as basic problems with applications in diverse areas. We describe here three different fields where these periodic latency problems occur. Recently, Studer [2008] described "rounding", a management process that can help to improve management and leadership skills. Studer believes that managers should make regular rounds to check on their employees. In that way, managers find out what matters to employees, and potential problems can be dealt with before they occur. This "rounding" model is based on the rounds doctors and nurses make to check on their patients in a hospital. Dimov et al. [2007] explore a method called "minirounds" that appears to improve physician-patient communication and satisfaction at a hospital. Minirounds are defined as follows: "A series of short patient encounters [each lasting about a 
minute] during which the physician asks patients about any changes in their condition and provide a concise daily update" (Dimov et al. [2007]). It is clear that efficiently organizing these mini-rounds is an instance of PLP. Notice that the latter application suggests a specific topology of the customers: Karuno et al. [1997] mention the tree network as being relevant for representing the corridor structure in hospitals and offices. We extensively study this topology in this work.

Another field where periodic latency problems occur is maintenance, more precisely preventive periodic maintenance. Machines, located at given positions (say different plants) need to be inspected regularly. Obviously, when a machine is viewed as a client, and when the periodicity of each machine is given, the PLP arises. Although there is quite some literature on preventive periodic maintenance, (see e.g. Dekker et al. [1997] for an overview), many contributions are stochastic, and we are not aware of deterministic situations where distances between machines are taken into account (see Anily et al. [1998] for a related problem).

A third field motivating PLP and PLPP concerns real-time task scheduling. In fact, periodic scheduling problems were already introduced in 1973 by Liu and Layland [1973]. They study the problem of scheduling periodic tasks with hard deadlines that coincide with the task periods. At that time, computers where used more and more to monitor and control industrial processes and the efficient scheduling of these control and monitor functions became important. Since then computers have evolved, more recent papers such as Bar-Noy et al. [2004] and Patil and Garg [2006], study periodic scheduling problems in the field of wireless devices. Here, in order to reduce power consumption, the goal is to find a periodic schedule such that wireless devices only need to be "awake" when they are being served. In this case a client is served regularly after a predefined amount of time (the period of the client), there is no travel time, each client, however, requires a service time.

\section{Related Problems}

Many routing and scheduling problems require a periodic solution. In the periodic TSP (Paletta [2002]), a set of customers each with a certain frequency is given. Each customer needs to be visited according to its frequency within a given planning period $T$. A solution then consists in a set of routes, one route for every day in the planning period $T$. This setting can be generalized to the periodic VRP, where more than one vehicle is available and several routes can be performed each day of the planning horizon $T$ (Mourgaya and Vanderbeck [2006]). These problems, however, do not belong to the class of latency problems.

Other applications of periodic scheduling problems can be found in scheduling of robotic cells, see e.g. Crama et al. [2000]; or in digital-signal-processing, see Verhaegh et al. [2001]. A signal processing algorithm consists in elementary operations that need to be carried out repeatedly on successive samples of a given digital signal. Korst et al. [1997] study the problem of nonpreemptively scheduling periodic tasks on a minimum number of identical processors. They mention an application where a number of continuous data streams must be read from a minimal number of identical disk units. Any situation where there is a repetitive execution of operations with strict timing requirements is relevant (Verhaegh et al. [2001]). In these settings, precedence constraints might also be 
present.

A non-periodic latency problem with profits is discussed in Coene and Spieksma [2008]. There, a profit $p_{i}$ is associated with every customer $i$ and these profits go down linearly with time, while the customer is waiting to be served. The goal is to select customers and to find a route for the server visiting these customers such that the collected profit is maximal. A similar problem with time windows is described by Frederickson and Wittman [2007]. Each service request is assigned to a time window and the goal is to find a tour that visits the maximum number of locations during their time windows. Each request that the repairman completes yields a given profit. These problems, however, are not periodic.

A problem that is probably closest to our setting is the problem described in Campbell and Hardin [2005]. In their problem, the number of servers needed to serve all customers is minimized, under the assumption that each customer $i \in N$ needs to be visited precisely every $q_{i}$ time-units. They show that a solution exists which is periodic (see Section 3) with length $T=\operatorname{lcm}\left(q_{1}, q_{2}, \ldots, q_{n}\right)$.

We give an overview of our results in Section 2. We elaborate on the issue of periodicity in Section 3. In Section 4 we deal with the single server problem, i.e., PLPP. In Section 5 we deal with PLP. The results of sections 4 and 5 can be generalized to a setting with an arbitrary given number of servers where the goal is to maximize the number of customers visited by these servers (the MPLPP), this is shown in Section 6. Finally, Section 7 discusses possible extensions.

\section{The results}

Figure 1 summarizes our results concerning the complexity of the PLPP for different settings of $p_{i}$ and $q_{i}$, in different metric spaces. From Theorem 3 and Corollary 2 it follows that in a general case with arbitrary profits and frequencies (d), the PLPP on the line and the PLPP on the circle are solvable in polynomial time. Hence, the same results hold for the more restricted topologies in (a), (b), and (c). Figure 1(a) shows results for the PLPP with unitary profits and a common frequency $Q$; this result is due to Theorem 7 and Corollary 3. Figure 1(b) represents the results for the PLPP with unitary profits and arbitrary frequencies; this follows from Theorem 5. Similarly, Figure 1(c) holds for PLPP with arbitrary profits and a common frequency $Q$, see Theorem 4 .

In the PLP, the goal is to minimize the number of servers needed to visit all customers. Profits are not applicable in this case. Results are represented in Figure 2 and follow directly from Theorem 8, Corollary 4, and Theorem 9. We see as our main contribution a complete classification of both PLPP and PLP for the following topologies: line, circle, star, tree, general; and we consider arbitrary profits $p_{i}$, versus $p_{i}=1$, and arbitrary periodicities $q_{i}$, versus $q_{i}=Q$, for all $i \in N$. We achieve this classification by either describing a polynomial time algorithm or giving an NP-hardness result. While some of these results are standard, others are not. In particular, the $O\left(n^{2}\right)$ algorithm for PLPP on the line and the $O\left(n^{5}\right)$ algorithm for PLP are not straightforward. 


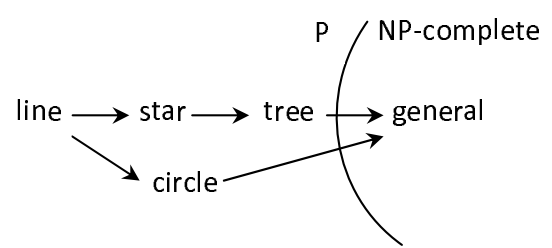

(a) PLPP with $p_{i}=1, q_{i}=Q$

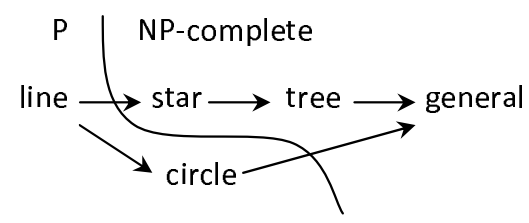

(c) PLPP with arbitrary $p_{i}, q_{i}=Q$

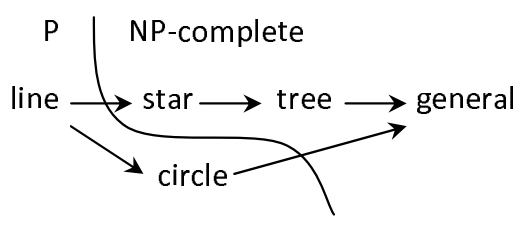

(b) PLPP with $p_{i}=1$, arbitrary $q_{i}$

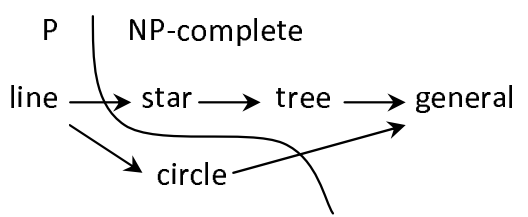

(d) PLPP with arbitrary $p_{i}$ and $q_{i}$

Figure 1: Complexity results PLPP

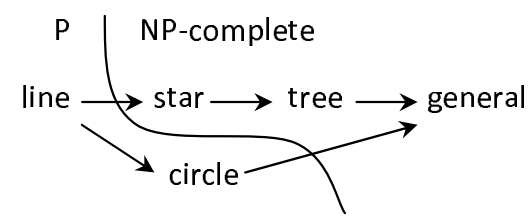

Figure 2: Complexity results PLP 


\section{Periodicity}

In this section periodicity of solutions is being analyzed. The analysis in this section is restricted to the PLPP. However, everything also holds for the PLP as a solution to the PLP consists in $s$ single server solutions, where $s$ denotes the number of servers used.

A solution to the PLPP can be represented by an infinite sequence of customers: customer $i$ appearing on the $p$-th position of the sequence indicates that the $p$-th customer visited is customer $i$. Let $L$ be a sequence representing a solution to the PLPP and $S$ the subset of customers visited. Notice that for $|S|=1$, periodicity is trivial.

We call any sequence of customers of finite length a subsequence.

Definition 1 A subsequence is called a $k$-cycle if

- the subsequence starts and ends with some customer $i \in N$ not appearing elsewhere in the subsequence, and

- each served customer $j \neq i$ appears with a frequency of at least 1 , and at most $k$, in the subsequence.

Definition 2 We say that a solution to PLPP is periodic if, when viewed after some position $p$, the sequence representing this solution is a concatenation of identical subsequences.

Definition 3 We say that a solution to PLPP is a $k$-cycle if, when viewed after some position $p$, the sequence representing this solution is a concatenation of identical $k$-cycles (where it holds that when two consecutive visits are performed to a single customer this can be considered a single visit to that customer).

Clearly, from the viewpoint of implementation and optimization, periodic solutions are preferable over non-periodic ones. Fortunately, we lose nothing by restricting the search for a solution to the class of periodic solutions:

Theorem 1 If there exists a feasible solution for an instance of PLPP that visits customer-set $S \subseteq N$, then there exists a periodic feasible solution visiting $S$.

Proof. Let $Q=\max _{i \in S} q_{i}$. All customers are positioned at distinct locations and $d_{i j} \geq 1$. Consider any feasible solution to PLPP, represented by a sequence $L$. Let us assume that the number of served customers is greater than $1,|S| \geq 2$. The proof relies on the following observation.

Observation $1 \mathrm{~L}$ consists of infinitely many $k$-cycles, for some $k$.

To argue that this observation holds, consider any position $p$ in the sequence $L$ and the customer $i$ visited at $p$. Then, at position $p+1$, a customer $j \neq i$ is visited and, at position $p+2$, either again customer $i$ is served or a "new" customer $l$. At a certain position $q>p,|S|-1$ different customers will have been served since position $p$. Thus, one customer, say customer $l$, will then necessarily be visited at a position $q^{\prime}>q$ and also at a position $p^{\prime}<p$; the total number of time units elapsed between these two visits can be no more 
than $Q$. It follows that between two consecutive visits to this customer $l$, all other customers are visited at least once. Since this holds for any position $p$, the observation is valid.

Now, as for a specific customer at most $n^{Q}$ different $k$-cycles exist, the total number of different $k$-cycles that can appear in $L$ cannot exceed $n^{Q+1}$.

Notice that we show here that the number of $k$-cycles is finite given that $d_{i j} \geq 1$; however, when $d_{i j}<1$ the number of $k$-cycles will be much larger but still finite. The observation above then implies that a same $k$-cycle will appear more than once. By repeating a subsequence that starts with this $k$-cycle, plus everything that followed this $k$-cycle up to its next appearance, we modify $L$ into a feasible solution that is periodic.

\section{The periodic latency problem with profits (PLPP)}

In this section, we prove the results summarized in Figure 1. Section 4.1 deals with the PLPP on the line or on a circle, Section 4.2 deals with the PLPP on a star, Section 4.3 deals with the PLPP on a tree, and finally, Section 4.4 deals with the PLPP on a arbitrary topology.

\subsection{PLPP on the line and the circle}

Consider first an instance of the PLPP with arbitrary profits $p_{i}$, and arbitrary periodicities $q_{i}$ where all customers are positioned on the line. We assume that $x_{1}<x_{2}<\ldots<x_{n}$, and we denote the distance between $x_{i}$ and $x_{j}$ as $d_{i j}$ for $i, j \in N$. We first show that for this special topology, we can restrict ourselves to solutions where the server simply oscillates between two customers. In other words, there is a solution that is a 2-cycle (see Definition 1).

Theorem 2 If there exists a feasible solution for a set of customers $S \subseteq N$, then there is a 2-cycle serving customer-set $S$, with $|S| \geq 2$.

Proof. Consider any pair of locations $x_{i}<x_{j}, i, j \in S$. Since $i, j \in S$, there exist two moments in time $t_{i}<t_{j}$ such that (i) the server is at $x_{i}$ at time $t_{i}$, (ii) the server is at $x_{j}$ at time $t_{j}$, and (iii) neither $x_{i}$ nor $x_{j}$ is visited at any time $t$ with $t_{i}<t<t_{j}$. Since $i \in S$ it must be true that $q_{i} \geq t_{j}-t_{i}+d_{j i} \geq d_{i j}+d_{j i}$. The first inequality is true because being able to serve both customers $i$ and $j$ implies that the periodicity of customer $i$ cannot be smaller than the time the server needs to travel from $x_{i}$ to $x_{j}$ and back (recall that we assume that the server travels at unit speed). Thus, the time needed to travel from $x_{i}$ to $x_{j}$ is at least equal to the distance $d_{i j}$, which gives us the second inequality. Similarly, we can argue that $q_{j} \geq d_{j i}+d_{i j}$. Thus, for all customers $i$ and $j$ in $S$ it holds that

$$
q_{i} \geq d_{i j}+d_{j i}
$$

and

$$
q_{j} \geq d_{j i}+d_{i j}
$$

We now exhibit a 2-cycle that is able to serve the customers in $S$. Indeed, consider a server that travels from the left-most customer in $S$ to the right-most customer in $S$, and back, and repeating this pattern. Each customer $i \in S$ is served as long as

$$
q_{i} \geq \max _{j \in S}\left(d_{i j}+d_{j i}\right)
$$


This, however, is implied by (1) and (2), and hence a 2-cycle serves the customers in $S$.

Using this claim it is not hard to argue that we can answer the question: can we visit all given customers? in $O(n)$ time. We refer to this problem as decisionPLPP.

Corollary 1 Decision-PLPP on the line can be answered in $O(n)$.

Proof. Given positions $x_{1}, \ldots, x_{n}$ we verify whether (3) holds. Given the line topology, the maximum in (3) can only be attained for $j=1$ or $j=n$. It follows that we need to verify $O(n)$ inequalities. $\square$

Theorem 3 PLPP on the line with arbitrary $p_{i}$ and arbitrary $q_{i}$ can be solved in $O\left(n^{2}\right)$.

Proof. We only need to search for a best 2-cycle (Theorem 2). Clearly, considering each possible combination of leftmost and rightmost customer, and then checking for all intermediate customers whether they can be served, yields an immediate $O\left(n^{3}\right)$ algorithm. We now proceed to describe an $O\left(n^{2}\right)$ algorithm.

Lemma 1 Consider a server traveling on the interval $\left[x_{i}, x_{i}+L\right]$; this server serves a customer $j$ if and only if

$$
\begin{aligned}
& \text { (i) } x_{i} \leq x_{j} \\
& \text { (ii) } x_{j} \leq x_{i}+L \\
& \text { (iii) } 2 L+2 x_{i}-2 x_{j} \leq q_{j} \\
& \text { (iv) } 2 x_{j}-2 x_{i} \leq q_{j} .
\end{aligned}
$$

Proof. Conditions (i) and (ii) state that point $x_{j}$ must be contained in the interval $\left[x_{i}, x_{i}+L\right]$. Conditions (iii) and (iv) follow from the following observation. The time needed for the server to travel from $x_{j}$ to the most right point of the interval and back to $x_{j}$ (i.e. twice the distance between $x_{j}$ and $x_{j}+L$ ), and the time needed to travel to the most left point of the interval and back (i.e. twice the distance between $x_{i}$ and $x_{j}$ ), respectively, may not be larger than the periodicity $q_{j}$.

According to Lemma 1, a customer $j$ is thus served by a server traveling in $\left[x_{i}, x_{i}+L\right]$ if and only if $x_{i} \leq x_{j}$ and $x_{i} \geq x_{j}-\frac{1}{2} q_{i}$ and if $L$ lies in the "activity interval"

$$
A_{j}:=\left[x_{j}-x_{i} ; x_{j}+\frac{1}{2} q_{j}-x_{i}\right] .
$$

The set of served customers $S(L)$ is then the following:

$$
S(L):=\left\{j: x_{i} \leq x_{j}, x_{i} \geq x_{j}-\frac{1}{2} q_{j}, \text { and } L \in A_{j}\right\} .
$$

Our algorithm consists of a preprocessing step and a main algorithm computing the best value for $L$, i.e. $L$ for which $\sum_{j \in S(L)} p_{j}$ is maximized, and this is done for every $i$.

(Preprocessing) For every customer $i$, let $l(i)=x_{i}$ and $r(i)=x_{i}+\frac{1}{2} q_{i}$. Sort all values of $l(i)$ and $r(i)$ in a global non-decreasing list $T$. 
(Main algorithm) For each customer $i$ the interval $\left[x_{i}, x_{i}+L\right]$ maximizing the resulting profit of the served customers can be computed as follows.

(i) For all customers in $T$, select the customers $j$ not violating conditions $x_{i} \leq x_{j}$ and $x_{i} \geq x_{j}-\frac{1}{2} q_{j}$ and define $l^{\prime}(j)=l(j)-x_{i}$ and $r^{\prime}(j)=$ $r(j)-x_{i} ; l^{\prime}(j)$ and $r^{\prime}(j)$ are then the leftmost and rightmost point, respectively, of the activity interval $A_{j}$ of customer $j$. Remark that the list containing all values $l^{\prime}(j)$ and $r^{\prime}(j)$ is sorted, we call this set $T^{\prime}$.

(ii) The first entry in $T^{\prime}$ will be $l^{\prime}(i)=0$, for the choice of $L=0$ the corresponding profit $P$ equals $p_{i}$. Set $P_{\max }=p_{i}$.

(iii) Work through $T^{\prime}$ and determine the profits for the corresponding values of $L$.

(a) If the next element in $T^{\prime}$ is a lower bound $l^{\prime}(j)$ of an activity interval $A_{j}$, set $P:=P+p_{j}$.

(b) If the next element in $T^{\prime}$ is an upper bound $r^{\prime}(j)$ of an activity interval $A_{j}$, set $P:=P-p_{j}$

(c) If $P>P_{\max }$, set $P_{\max }:=P$.

Select the highest $P_{\max }$ over all customers $i$.

The preprocessing step takes $O(n \log n)$, the main algorithm has $O(n)$ iterations and each iteration takes $O(n)$, yielding a total complexity of $O\left(n^{2}\right)$.

Notice that Theorem 3 is also valid for a server whose speed differs between traveling to the right and traveling to the left.

Consider now an instance of PLP where all customers are positioned on a circle: we can simply extend Theorem 3 to this case:

Corollary 2 PLPP on the circle with arbitrary $p_{i}$, and arbitrary $q_{i}$ can be solved in $O\left(n^{2}\right)$.

Proof. A solution to PLPP on the circle is either a 2-cycle or the full circle (which is actually a 1-cycle). The best 2-cycle can be found using the same algorithm as described for the line. When fixing a leftmost customer, the rightmost customer is found going clockwise through the circle. Thus, the optimal solution to an instance of PLPP on the circle can be found in $O\left(n^{2}\right)$

\subsection{PLPP on a star}

Let us consider a star graph (called a star). The customers are positioned in the end nodes and, in addition to a profit $p_{i}$ and a periodicity $q_{i}$, there is a distance $d_{i}$ given which denotes the distance between the position of customer $i$ and the center of the star, $i \in N$

It is easy to see that the PLPP where all $p_{i}=1$ and $q_{i}=Q$ for all $i \in N$ is solvable in polynomial time. Indeed, by selecting customers with the smallest $d_{i}$ until the tour length exceeds $Q$, an optimal solution is found. In fact, a more general result is shown in Section 4.3. In Section 5 we show that adding a second server already makes the problem NP-hard.

When profits $p_{i}$ are arbitrary, and $q_{i}=Q$, PLPP on a star can be shown to be equivalent to the knapsack problem. 
Theorem 4 PLPP on a star with arbitrary profits $p_{i}$, and with all $q_{i}=Q$, is NP-hard.

Proof. We reduce from knapsack, which is a weakly NP-hard problem Garey and Johnson [1979]. Consider an instance of the knapsack problem with $n$ items where each item $i$ has a certain value $w_{i}$ and a requirement $a_{i}$. The knapsack has size $B$ and the question is whether we can fit a subset of the items with a total value of $W$ in this knapsack.

Now construct an instance of PLPP on a star as follows. There are $n$ customers. Each customer $i \in N$ has an associated weight $p_{i}:=w_{i}$, a periodicity $Q:=B$, and a distance to the center of the star $d_{i}:=\frac{1}{2} a_{i}$. Does there exist a subset of the customers with total profit equal to $W$ such that each customer is visited at least once within each time period $Q$ ?

In case the instance of knapsack is a yes-instance, we can copy that solution to the instance of PLPP: selected items correspond to selected customers. Starting in the center, it is clear that by visiting the selected customers in any order, and repeating that pattern gives a feasible solution. When the instance of PLPP admits a yes, there is a set of customers that we can apparently serve. A served customer $i$ implies a travel time of $2 d_{i}=a_{i}$. Feasibility of the PLPPinstance ensures that the corresponding set of items fits in the knapsack.

When we consider PLPP on a star with $p_{i}=1$, and arbitrary periodicities $q_{i}$, the problem becomes NP-hard.

Theorem 5 PLPP on a star with all $p_{i}=1$, and arbitrary periodicities $q_{i}$, is NP-hard.

Proof. We show that 3-Partition can be reduced to PLPP on a star with $p_{i}=1$, and arbitrary periodicities $q_{i}$. This proof is based on the proof of Korst et al. [1997] for NP-hardness of Scheduling Periodic Tasks with Slack (PSSP).

An instance of 3 -Partition consists of a set $A$ with $3 m$ items and a positive integer $B$ representing the size of the $m$ bins. Each item $a_{i} \in A$ has an associated size $z_{i}$ for which it holds that $\frac{B}{4}<z_{i}<\frac{B}{2}$ and $\sum_{a_{i} \in A} z_{i}=m B$. Can $A$ be packed into $m$ bins, each containing three items?

We construct an instance of our special case of PLPP on a star such that the items can be packed in $m$ bins if the customers in the corresponding PLPP, each with their respective periodicity, can be served by a single server. We are given a set of $n:=3 m+1$ customers, each with periodicity $q_{i}:=m(B+2)$ and distance $d_{i}:=\frac{1}{2} z_{i}$ to the center of the star, for $i=1, \ldots, 3 m$. Further, customer $3 m+1$ has $q_{3 m+1}:=B+2$ and $d_{3 m+1}:=1$. The question is whether there is a solution serving all $3 m+1$ customers.

If 3-Partition has a solution, it is clear how to copy that solution to the PLPP instance, and get a solution serving all $3 m+1$ customers. If the PLPP instance has a solution in which all $3 m+1$ customers are served, then, between two consecutive visits to customer $3 m+1$, there are exactly $B$ time units left that can be used to visit other customers. Each of the $3 m$ customers left must be visited at least once in time period $m(B+2)$. In that time period there are $m$ available time slots of $B$ time units. Thus, the $3 m$ customers can be assigned to the $m$ different time slots if and only if the corresponding items can be packed in $m$ bins. $\square$ 
The following holds when periods are arbitrary and distances unitary:

Theorem 6 PLPP on a star with unitary distances, unitary profits, and arbitrary periodicities is solvable in polynomial time.

Proof. Consider an optimal solution visiting a maximal subset $S$ of customers. Set $T$ is the set of unvisited customers, $N=S+T$. It holds that for any two customers $i \in T$ and $j \in S$ with $q_{i}>q_{j}, i$ can be moved to subset $T$ and $j$ to subset $S$. A new optimal solution is obtained. Thus, an optimal solution can easily be found by selecting customers in decreasing order of $q$ until a feasible solution is no longer obtained.

\subsection{PLPP on a tree}

We argue here that PLPP on a tree with $p_{i}=1$ and $q_{i}=Q$ is nothing but an orienteering problem (OP). Coene et al. [2008] describe how to modify an algorithm from Johnson and Niemi [1983] in order to solve the orienteering problem restricted to a tree. An instance of $\mathrm{OP}$ on a tree consists of a set of vertices $N$ where each vertex $i \in N$ has an associated profit $p_{i}$ and each edge between two vertices $i$ and $j$ in the tree has a cost $c_{i j}$. A maximum on the cost $C$ is given. The goal is to find a route visiting a subset of the vertices with cost no more than $C$ and collecting a maximal amount of profit. An instance of PLPP with $p_{i}=1$ and $q_{i}=Q$ is then an orienteering problem with $p_{i}=1$ $(\forall i \in N), c_{i j}:=d_{i j}$, and with $C:=Q$. The algorithm in Coene et al. [2008] solves OP on a tree in $O\left(n P_{t o t}\right)$, with $P_{t o t}$ the sum of all given profits; since $p_{i}=1, \forall i \in N$, total running time for PLPP is only $O\left(n^{2}\right)$.

Corollary 3 PLPP on a tree with $p_{i}=1$ and $q_{i}=Q$ is solvable in $O\left(n^{2}\right)$.

\subsection{PLPP on an arbitrary topology}

Theorem 7 PLPP with $p_{i}=1$, and $q_{i}=Q$, is NP-hard.

Proof. We prove NP-completeness of this variant of PLPP by a reduction from Hamiltonian cycle. An instance of the Hamiltonian cycle problem is specified as follows: given a graph $G=(V, E)$, does there exist a Hamiltonian cycle in $G$ ?

Now consider the following instance of PLPP. A node in $V$ corresponds to a customer, and we set $n:=|V|$. For each pair of customers $i, j$ that is connected via an edge in $E$ we set $d_{i j}:=1$, else we set $d_{i j}:=2$. The periodicity is $q_{i}:=|V|$ for each $i \in N$. Now, does there exist a solution to PLPP with value $n$ ?

If a Hamiltonian cycle exists in $G$, there exists a tour in the PLPP instance with length $n$ visiting all the locations. This solution is periodic. Vice versa, if a solution serving all customers in the PLPP instance exists, $G$ must contain a Hamiltonian cycle.

\section{The periodic latency problem (PLP)}

In this section we study the PLP and we prove the results summarized by Figure 2. In the PLP our goal is to minimize the number of servers needed to visit all 
customers. We assume that every customer must be assigned to and served by a single server; this is a crucial assumption as can be seen from the example in Figure 3. Observe that when each customer must be served by one server, three servers are needed to serve the customers in the example. If, however, that assumption is dropped, the three customers from the example can be served using only 2 servers, each server alternatingly serving the middle customer.

\begin{tabular}{ccccc}
$q_{1}=2$ & \multicolumn{1}{c}{$x_{2}$} & & $x_{3}$ \\
& 1 & & 1 & \\
$q_{1}$ & $q_{2}=1$ & & $q_{3}=2$
\end{tabular}

Figure 3: PLP

Determining for an arbitrary topology, whether a single server suffices to serve all customers, is NP-hard; this follows directly from Theorem 7 . In this section we show that a dynamic programming approach solves the case of the line in polynomial time, whereas PLP on a star remains NP-hard.

\subsection{PLP on the line}

We develop a dynamic programming algorithm for the PLP on the line. Given are $n$ customers with their positions $x_{1}<x_{2}<\ldots<x_{n}$ on the line; and a set of $n$ identical servers. The goal is to minimize the number of servers necessary to visit all customers periodically, i.e. without violating any $q_{i}$.

There exists an optimal schedule to an instance of PLP on the line of the following shape:

- Every server $s$ commutes between the left and the right endpoint of its interval $I_{s}$.

- The intervals $I_{s}$ and $I_{t}$ of any two different servers $s$ and $t$ are either disjoint, or one of them does contain the other one.

- The left endpoint of interval $I_{s}$ is only assigned to server $s$.

We now describe an informal argument establishing the second property described above. Suppose that every optimal solution contains two intervals $I_{s}$ and $I_{t}$ that are disjoint nor contained. Then, a client $i$ at position $x_{i}$ exists, contained in both intervals, which is served by one of the servers, say server $t$, and cannot be served by server $s$. Define $x_{s, l}$ and $x_{s, r}$ as the leftmost and rightmost point, respectively, of $I_{s}$ and analogue $x_{t, l}$ and $x_{t, r}$ for $I_{t}$. We can assume then without loss of generality that $x_{s, l} \leq x_{t, l} \leq x_{i} \leq x_{s, r} \leq x_{t, r}$. As $x_{i}$ is served by server $t$ and cannot be served by $s$, it must hold that $d_{x_{s, l}, x_{i}} \geq$ $d_{x_{t, r}, x_{i}}$. As a result all customers positioned on the interval $\left[x_{i}, x_{s, r}\right]$ and served by server $s$ can also be served by server $t$, yielding two disjoint intervals $I_{s}$ and $I_{t}$.

We say that in some schedule a server covers customer $i$ if the time elapsed between two consecutive visits of this server to the customers is at most $q_{i}$. The following lemma clearly holds: 
Lemma 2 Let $a \leq b \leq c \leq d$ be four request points. Assume that server $s$ commutes on the outer interval $[a, d]$, and that server $t$ commutes on the inner interval $[b, c]$. Then every point in interval $[b, c]$ that is covered by the outer server s will also be covered by the inner server $t$.

Indeed, the frequency with which any point $x_{i}$ within $[b, c]$ is visited by the server commuting in $[b, c]$ is higher than for the server commuting in $[a, d]$.

\section{A dynamic programming algorithm}

The dynamic program is built around the following definition.

Definition 4 Let $i$ and $j$ be integers with $1 \leq i, j \leq n$. Let $i^{\prime}$ and $j^{\prime}$ be integers such that either (i) $1 \leq i^{\prime} \leq j^{\prime} \leq n$ and $x_{1} \leq x_{i^{\prime}} \leq x_{j^{\prime}} \leq x_{n}$ where $x_{i}$ and $x_{j}$ are contained in the interval $\left[x_{i^{\prime}}, x_{j^{\prime}}\right]$, or such that $(i i) i^{\prime}=j^{\prime}=0$.

Then $F\left[i ; j ; i^{\prime} ; j^{\prime}\right]$ denotes the smallest number of servers that can serve all customers $k=i, i+1, \ldots, j$ with the exception of the customers that are already covered by an external server that commutes on the interval $\left[x_{i^{\prime}}, x_{j^{\prime}}\right]$. (In the case $i^{\prime}=j^{\prime}=0$, there is no such external server.)

Notice that in this definition we explicitly allow the situation $j<i$, which yields an empty interval and an empty set of requests. We will now compute all the values $F\left[i ; j ; i^{\prime} ; j^{\prime}\right]$ step by step and in increasing order of $j-i$ (the number of customers in the underlying interval).

All cases with $j<i$ have empty intervals, and hence need $F\left[i ; j ; i^{\prime} ; j^{\prime}\right]=0$ servers. In the remaining cases we have $j \geq i$. If the leftmost point is already served by the external server, we may simply set $F\left[i ; j ; i^{\prime} ; j^{\prime}\right]:=F\left[i+1 ; j ; i^{\prime} ; j^{\prime}\right]$. Otherwise the leftmost point $i$ must be served by some new server $s$. We branch into several cases that depend on the behavior of $s$.

Assume that the server $s$ commutes on the interval $I_{s}=\left[x_{i}, x_{l}\right]$ with $i \leq l \leq$ $j$. Then we need at least

$$
\alpha(l):=F[i+1 ; l ; i ; l]
$$

additional servers working and serving in interval $I_{s}$. Because of Lemma 2 , the influence of the external server commuting on the outer interval $\left[x_{i^{\prime}}, x_{j^{\prime}}\right]$ has now become irrelevant; everything this server has covered will also be covered by the new server $s$. Furthermore, we need at least

$$
\beta(l):=F\left[l+1 ; j ; i^{\prime} ; j^{\prime}\right]
$$

servers working in the remaining uncovered interval $\left[x_{l+1}, x_{j}\right]$. Therefore the smallest possible number of servers in this situation is

$$
F\left[i ; j ; i^{\prime} ; j^{\prime}\right]=\min \{1+\alpha(l)+\beta(l) \mid i \leq l \leq j\} .
$$

In the end, the answer to the global problem can be found in $F[1 ; n ; 0 ; 0]$. We have to compute $O\left(n^{4}\right)$ values $F\left[i ; j ; i^{\prime} ; j^{\prime}\right]$, and the computation of each value takes linear time. Hence the overall running time is $O\left(n^{5}\right)$.

Theorem 8 PLP on the line can be solved in $O\left(n^{5}\right)$. 


\subsection{PLP on a circle}

The algorithm for the problem on the line can be extended to the circle, adding a factor $n$ to the running time of the line algorithm.

Corollary 4 PLP on the circle can be solved in $O\left(n^{6}\right)$.

Proof. PLP on a circle can be broken down to $n$ instances of the problem on the line. Solving all these line instances and selecting the best from these $n$ solutions, yields an optimal solution to the PLP on the circle. Thus, the optimal solution to an instance of PLP on the circle can be found in $O\left(n^{6}\right)$.

\subsection{PLP on a tree}

We prove that the PLP on a star with $q_{i}=Q$ is NP-hard; NP-hardness of the PLP on a tree with arbitrary $q_{i}$ follows immediately from this result. In Section 4.2 it was shown that the periodic latency problem on a star graph with all customers having the same profit and requiring equal frequency is easy to solve; adding a second server though makes the problem much harder to solve.

Theorem 9 PLP on star is NP-hard, even if $q_{i}=Q$, for all $i \in N$.

Proof. We reduce from partition. An instance of the partition problem has a set $X=\left\{x_{1}, \ldots, x_{n}\right\}$ with $\sum_{i=1}^{n} s\left(x_{i}\right)=2 k$, where $s\left(x_{i}\right)$ denotes the size of $x_{i}$. Can the set $X$ be partitioned into two sets $X_{1}$ and $X_{2}$ such that $\sum_{x \in X_{1}} s(x)=$ $\sum_{x \in X_{2}} s(x)=k$ and each element occurs exactly once?

An instance of PLP on a star is constructed as follows. There is a star with $n$ spokes, and a customer located at each spoke with distance $\frac{1}{2} x_{i}$ from the center of the spoke, for $i=1, \ldots, n$. Each customer (spoke) has a periodicity $Q:=k$, meaning that the maximal time that can pass between two consecutive visits to a customer equals $k$. There are two servers, traveling at unit speed, positioned in the center of the graph. Now, does there exist a route for each of the servers such that all customers can be served periodically?

If a solution to partition exists, the set of customers in $X_{1}$ can be assigned to one server and the customers in $X_{2}$ to the other server and each server can visit these customers in $Q$ time units. If a solution to PLP on the star exists, each customer is visited once within every $Q$ time units. Since total travel time to visit all customers equals $2 Q$, it must be the case that every server travels exactly $Q$ time units. Customers visited by server 1 can be assigned to one set and customers visited by server 2 to the second set, and a solution for partition is obtained.

As a result, PLP on a tree is NP-hard.

\section{The case of multiple servers: MPLPP}

The complexity results from the previous section can be extended to a periodic latency problem with profits and multiple servers, denoted by MPLPP. As before, we are given a set of $n$ customers with their positions $x_{1}, x_{2}, \ldots, x_{n}$; and each customer $i$ has an associated profit $p_{i}$ and periodicity $q_{i}$. Further, $S$ identical servers are given, with $S<n$. (The case $S=1$ is dealt with in Section 4). 
The goal is then to find a repeatable route for each server collecting a maximal amount of profit without violating the $q_{i}$ 's. We show that, when all costumers are positioned on the line/circle the dynamic programming algorithm from the previous section can be transformed to solve the MPLPP in polynomial time. On a tree or a arbitrary graph the problem is NP-hard. One can easily check that Lemma 2 still holds for MPLPP on the line. Then a state in the dynamic programming algorithm is defined as follows.

Definition 5 Let $i$ and $j$ be integers with $1 \leq i, j \leq n$. Let $i^{\prime}$ and $j^{\prime}$ be integers such that either (i) $1 \leq i^{\prime} \leq j^{\prime} \leq n$ and $x_{1} \leq x_{i^{\prime}} \leq x_{j^{\prime}} \leq x_{n}$ where $x_{i}$ and $x_{j}$ are contained in the interval $\left[x_{i^{\prime}}, x_{j^{\prime}}\right]$, or such that $(i i) i^{\prime}=j^{\prime}=0$.

Then $P\left[i ; j ; i^{\prime} ; j^{\prime} ; s\right]$ denotes the maximum profit that can be collected serving customers in the interval $\left[x_{i}, x_{j}\right]$ using $s \leq S$ servers, excluding the customers that are already covered by an external server that commutes on the interval $\left[x_{i^{\prime}}, x_{j^{\prime}}\right]$. (In the case $i^{\prime}=j^{\prime}=0$, there is no such external server.)

Now, we compute all the values of $P\left[i ; j ; i^{\prime} ; j^{\prime} ; s\right]$. All cases with $j<i$ have empty intervals, and hence yield a profit $P\left[i ; j ; i^{\prime} ; j^{\prime} ; s\right]=0$. When $j \geq i$ we can distinct several cases. If the leftmost point $i$ is covered by an external server or is not served at all, it holds that $P\left[i ; j ; i^{\prime} ; j^{\prime} ; s\right]=P\left[i+1 ; j ; i^{\prime} ; j^{\prime} ; s\right]$. Otherwise $i$ is served by a server from $s$, say $s_{1}$, traveling on the interval $\left[x_{i}, x_{l}\right]$ with $i \leq l \leq j$. Total profit for this state then consists of three parts: (i) $p(l)=\sum_{i \in S\left(s_{1}\right)} p_{i}$, the profit of the set of customers $S\left(s_{1}\right)$ covered by $s_{1}$; (ii) $\alpha\left(l, s^{\prime}\right)=P\left[i+1, l, i, l, s^{\prime}\right]$, the profit of the servers traveling within the interval covered by $s_{1}$; and (iii) $\beta\left(l, s-s^{\prime}\right)=P\left[l+1, j, i^{\prime}, j^{\prime}, s-s^{\prime}-1\right]$, the profit that can be collected in the remaining interval by the remaining servers. The optimal value is then:

$$
P\left[i ; j ; i^{\prime} ; j^{\prime} ; s\right]=\max _{l, s^{\prime}}\left\{p(l)+\alpha\left(l, s^{\prime}\right)+\beta\left(l, s^{\prime}\right) \mid i \leq l \leq j, 0 \leq s^{\prime} \leq s\right\} .
$$

The state $P[i ; j ; 0 ; 0 ; S]$ yielding maximal profit gives the optimal solution. There are $O\left(n^{4} S\right)$ states to be computed and each state requires $O\left(n^{2} S\right)$ time. Overall running time is then $O\left(n^{6} S^{2}\right)$.

Theorem 10 MPLPP on the line can be solved in $O\left(n^{6} S^{2}\right)$.

As in the previous section, the algorithm for MPLPP on the line can be extended adding a factor $n$ to the running time.

Corollary 5 MPLPP on the circle can be solved in $O\left(n^{7} S^{2}\right)$.

$N P$-hardness of MPLPP on a tree follows from the fact that MPLPP on a star is $N P$-hard.

Theorem 11 MPLPP on a star is $N P$-hard, even if all $q_{i}=Q$.

Proof. It is easy to see that the proof of Theorem 9 is directly applicable to this problem.

It follows that the problem is NP-hard on arbitrary graphs.

\section{Conclusion}

We were able to settle complexity of a number of variants of the PLPP and the PLP. Our results still hold when customers are weighted, as a customer will 
always be served when a server passes by. However, some interesting questions remain. It would, for instance, be interesting to study the influence of repair times on complexity. This seems to make the problems much harder. Indeed, when repair times are added for the customers, Lemma 2, which is crucial for the dynamic programming algorithm, no longer holds. Further, it is also not clear what happens when servers have restricted capacity. And what if servers are not identical, meaning that they travel at different speeds or with different operating costs?

\section{Epilogue}

In the Treaty of Verdun of 843 the three grandsons of Charlemagne divided the empire into three kingdoms. Hence thirty years after Charlemagnes death, one needed already three servers to guarantee the loyalty of all the counts...

\section{References}

S. Anily, C. A. Glass, and R. Hassin. The scheduling of maintenance service. Discrete Applied Mathematics, 82:27-42, 1998.

A. Bar-Noy, V. Dreizin, and B. Patt-Shamir. Efficient algorithms for periodic scheduling. Computer Networks, 45:155-173, 2004.

A. M. Campbell and J. R. Hardin. Vehicle minimization for periodic deliveries. European Journal of Operational Research, 165:668-684, 2005.

S. Coene and F. C. R. Spieksma. Profit-based latency problems on the line. Operations Research Letters, 36:333-337, 2008.

S. Coene, C. Filippi, F. C. R. Spieksma, and E. Stevanato. The traveling salesman problem on trees: balancing profits and costs. Submitted, 2008.

Y. Crama, V. Kats, J. van de Klundert, and E. Levner. Cyclic scheduling in robotic flowshops. Annals of Operations Research, 96:97-124, 2000.

W. E. de Paepe, J. K. Lenstra, J. Sgall, R. A. Sitters, and L. Stougie. Computeraided compexity classification of dial-a-ride problems. INFORMS Journal on Computing, 16:120-132, 2004.

R. Dekker, F. A. van der Duyn Schouten, and R. E. Wildeman. A review of multi-component maintenance models with economic dependence. Mathematical Methods of Operations Research, 45:411435, 1997.

V. Dimov, A. Kumar, R. Hebbar, W. Fares, and P. Sharma. Mini-rounds improve pphysician-patient communication and satisfaction (abstract). Journal of Hospital Medicine, SHM Annual Meeting, 2:48, 2007.

G. N. Frederickson and B. Wittman. Approximation algorithms for the traveling repairman and speeding deliveryman problems with unit-time windows. APPROX and RANDOM 2007, Lecture Notes in Computer Science (LNCS), 4627:119-133, 2007. 
M. R. Garey and D. S. Johnson. Computers and Intractability: A Guide to the Theory of NP-completeness. Freeman, San Francisco, 1979.

D. S. Johnson and K. A. Niemi. On knapsacks, partitions, and a new dynamic programming technique for trees. Mathematics of Operations Research, 8: 1-14, 1983.

Y. Karuno, H. Nagamochi, and T. Ibaraki. Vehicle scheduling on a tree with release and handling times. Annals of Operations Research, 69:193-207, 1997.

J. Korst, E. Aarts, and J. K. Lenstra. Scheduling periodic tasks with slack. INFORMS Journal on Computing, 9:351-362, 1997.

C. L. Liu and J. W. Layland. Scheduling algorithms for multiprogramming in a hard-real-time environment. Journal of the Association for Computing Machinery, 20:46-61, 1973.

M. Mourgaya and F. Vanderbeck. Problème de tournées de véhicules multipériodiques: Classification et heuristique pour la planification tactique. RAIRO Operations Research, 40:169-194, 2006.

Giuseppe Paletta. The period traveling salesman problem: a new heuristic algorithm. Computers and Operations Research, 29(10):1343-1352, 2002. ISSN 0305-0548. doi: http://dx.doi.org/10.1016/S0305-0548(01)00035-1.

S. Patil and V. K. Garg. Adaptive general perfectly periodic scheduling. Information Processing Letters, 98:107-114, 2006.

Q. Studer. Results that last: hardwiring behaviors that will take your company to the top. John Wiley \& Sons, Inc., Hoboken, New Jersey, 2008.

W. F. J. Verhaegh, E. H. L. Aarts, P. C. N. van Gorp, and P. E. R. Lippens. A two-stage solution approach to multidimensional periodic scheduling. IEEE Transactions on computer-aided design of integrated circuits and systems, 20: 1185-1199, 2001. 